\title{
The Relationship between Masticatory Function and Dysphagia in Patients with Myotonic Dystrophy
}

\author{
George Umemoto, DDS, PhD, ${ }^{a}$ Hirokazu Nakamura, DDS, PhD, ${ }^{b}$ \\ Yoshihiro Tsukiyama, DDS, PhD, ${ }^{c}$ Kiyoshi Koyano, DDS, PhD, ${ }^{c}$ \\ and Toshihiro Kikuta, DDS, PhD \\ ${ }^{a}$ Department of Oral and Maxillofacial Surgery, Faculty of Medicine, Fukuoka University, Fukuoka, Japan \\ ${ }^{\mathrm{b}}$ Dental Branch, National Center of Neurology and Psychiatry, Musashi Hospital, Tokyo, Japan \\ ${ }^{c}$ Department of Removable Prosthodontics, Faculty of Dental Science, Kyushu University, Fukuoka, Japan
}

\section{Clinical significance}

This study analyzes the relationship between masticatory function and dysphagia in patients with myotonic dystrophy. The results suggest that masticatory disturbances should be considered in myotonic dystrophy patients with dysphagia.

\begin{abstract}
Purpose: This study aims to investigate the relationship between masticatory function and dysphagia in patients with myotonic dystrophy (MyD).

Methods: Eighteen MyD patients with an average age of 54.1 years (SD, 5.7 years) were enrolled as subjects of this study. They were divided into 2 groups: a non-dysphagia group (12 patients) that consumed ordinary rice along with an ordinary supplementary diet and a dysphagia group (6 patients) that consumed semisolid rice along with a chopped supplementary diet. The maximum bite force, activity in the temporal and masseter muscles during 1 chewing cycle, number of food items that were considered as easy to chew, and occlusal contact area were investigated, and the proportion of patients with anterior open bite was analyzed. The results obtained were compared between the groups.

Results: The maximum bite force in the dysphagia group was statistically much smaller than that in the non-dysphagia group $(P=0.024)$. On the other hand, the masticatory muscle activity in the dysphagia group was statistically much greater than that in the non-dysphagia group $(P=0.048)$. The proportion of $\mathrm{pa}^{-}$ tients with anterior open bite in the dysphagia group was higher than that in the non-dysphagia group.
\end{abstract}

Conclusion: These results suggest that the bite force

\section{Corresponding to: Dr George Umemoto}

Department of Oral and Maxillofacial Surgery, Faculty of

Medicine, Fukuoka University

7-45-1 Nanakuma, Jonan-ku, Fukuoka 814-0180, Japan

Tel: +81-92-801-1011, Fax: +81-92-801-1044

E-mail: george@minf.med.fukuoka-u.ac.jp

Received on December 27, 2006 / Accepted on February 28, 2007 and anterior open bite might influence dysphagia in MyD patients; however, this remains a speculation. It is necessary to consider masticatory disturbances in MyD patients with dysphagia.

Key words: myotonic dystrophy, masticatory function, dysphagia, anterior open bite, bite force

\section{Introduction}

Myotonic dystrophy (MyD) is described as a myopathy of autosomal dominant inheritance that is characterized by muscle atrophy, myotonia, and multisystem diseases. ${ }^{1}$ Some reports have indicated a high incidence of dental problems such as caries and periodontitis, aberrations of craniofacial morphology, malocclusion, malalignment, a reduced masticatory muscle function, dysmasesis, dysphagia, poor oral hygiene, and dislocation of the temporomandibular joint in cases of $\mathrm{MyD}^{2,3}$ Previous reports ${ }^{4,5}$ have indicated that MyD patients find some foods items difficult to chew because of their weak bite force and that they compensate for this weakness by considerably increasing their masticatory muscle activity while chewing. In clinical practice, the attending physicians selected a diet type for each patient after considering the dysphagia status. However, masticatory disturbances also appear to influence dysphagia. In this study, to investigate the relationship between the masticatory function and dysphagia in $\mathrm{MyD}$ patients, the masticatory function was compared between the dysphagia and non-dysphagia groups. 


\section{Materials and methods}

\section{Subjects}

Eighteen inpatients (11 men and 7 women) with MyD who were treated at the Department of Neurology, National Center of Neurology and Psychiatry, Musashi Hospital, between January 1999 and December 2002 were enrolled in the study. They all maintained the vertical stop despite some of the lost molars or premolars. Inpatients who were not referred to the Dental Branch and outpatients were excluded from the study. The average age of the patients was 54.1 years (SD, 4.7 years). Anterior open bite was exhibited by 8 of the 18 patients. Based on the medical records, the patients were divided into 2 groups: a non-dysphagia group and a dysphagia group. The attending physicians selected a diet type for each patient after considering their dysphagia status.

\section{Non-dysphagia group}

This group comprised $12 \mathrm{MyD}$ patients (6 men and 6 women) who consumed ordinary rice along with an ordinary supplementary diet. The average age of these patients was 54.0 years (SD, 5.7 years).

\section{Dysphagia group}

This group comprised $6 \mathrm{MyD}$ patients (5 men and 1 woman) who consumed semisolid rice along with a chopped supplementary diet. The average age of these patients was 53.8 years (SD, 1.8 years).

The patients were informed about the risks involved in the examinations and were clearly explained that they could voluntarily withdraw from the study at any time. Informed consent was obtained from all the patients prior to their participation. The same author (G.U.) performed all of the following examinations.

\section{Maximum bite force}

The maximum bite force on each side was recorded at the right and left first molar regions by using an Occlusal Force Meter GM10 ${ }^{6}$ (Nagano Keiki, Co., Tokyo, Japan). Data were acquired in triplicate at each region, and an average was determined.

\section{Muscle activity}

The muscle activities in the temporal and masseter muscles during 1 chewing cycle were mea- sured using a K6I measuring system ${ }^{7}$ (Myotronics Inc., Seattle, USA). The electrical activities in the masseter and temporal muscles were recorded using bipolar surface electrodes. To prevent the risk of aspiration, the subjects were instructed to chew a cotton roll that cannot be aspirated like food. The chewing movements were recorded over a period of approximately 15 seconds, and the data obtained for 5 chewing strokes, i.e., from the sixth to the tenth stroke, were analyzed. The electrical activity during each individual stroke was converted into percent electrical activity that was attained during maximum voluntary teeth clenching (MVC\%).

\section{Number of food items that were considered as easy to chew}

A questionnaire ${ }^{8}$ (Sato, 1989) survey of the patients was conducted to determine the ease with which the patients could chew 20 types of food items listed in the questionnaire, and the number of food items that could be chewed easily was determined. The following 20 food items were included in the questionnaire: bean curd (tofu), boiled eggplants, boiled potatoes, boiled carrots, artificially grown soybean, boiled fish paste (kamaboko), potato chips, burdock, rice cake cubes, beefsteak, peanuts, pickled radish, hard biscuits, hard crackers, hard pickled radish, fresh ear shell, dried cuttlefish, dried shell ligament, a chewing gum, and a whole apple.

\section{Occlusal contact area}

The occlusal contact area was measured using a Dental Prescale System ${ }^{9}$ (Fuji Film, Tokyo, Japan). This method uses a sheet that undergoes a color-developing chemical reaction to detect the contact area, and a computerized analysis system to evaluate the balance of the occlusal load. Each subject was instructed to bite the Dental Prescale sheet (Dental Prescale 50H R-type; Fuji Film, Tokyo, Japan) with maximum force for 3 seconds; this test was repeated 3 times. The average result of the test conducted in triplicate was obtained.

\section{Data analysis}

Student's $t$ test was conducted to compare the values of maximum bite force, muscle activity, and occlusal contact area between the dysphagia and non-dysphagia groups. Mann-Whitney's U test was conducted to compare the number of food items that were considered as easy to chew 
Table 1 Differences in masticatory function between the non-dysphagia and dysphagia groups.

\begin{tabular}{|c|c|c|c|c|c|}
\hline & \multicolumn{2}{|c|}{ Non-dysphagia group } & \multicolumn{3}{|c|}{ Dysphagia group } \\
\hline & $\mathrm{X}$ & SD & $\mathrm{X}$ & $\mathrm{SD}$ & $\mathrm{p}$ \\
\hline Maximum bite force $(\mathrm{N})$ & 86.2 & 36.3 & 46.1 & 19.6 & $<0.05$ \\
\hline Muscle activity (MVC\%) & 33.6 & 15.6 & 52.0 & 20.8 & $<0.05$ \\
\hline Occlusal contact area $\left(\mathrm{mm}^{2}\right)$ & 7.4 & 5.2 & 5.4 & 7.0 & n.s. \\
\hline $\begin{array}{l}\text { Number of foods that were } \\
\text { considered as easy to chew }\end{array}$ & 14.5 & 2.2 & 12.7 & 2.3 & n.s. \\
\hline
\end{tabular}

p, statistically significant level; n.s., not statistically significant.

Table 2 Proportion of patients with anterior open bite.

\begin{tabular}{lcc}
\hline & $\begin{array}{c}\text { Non-dysphagia group } \\
\text { n/all patients }(\%)\end{array}$ & $\begin{array}{c}\text { Dysphagia group } \\
\text { n/all patients }(\%)\end{array}$ \\
\hline Anterior open bite & $4 / 12(33.3 \%)$ & $4 / 6(66.7 \%)$ \\
\hline
\end{tabular}

between the 2 groups. With regard to the ratio of the patients with anterior open bite to all patients in the 2 groups, no statistical analysis was conducted because of very few subjects.

All statistical analyses were performed using SPSS $13.0 \mathrm{~J}$ for Windows.

\section{Results}

Of the 4 factors investigated, differences were observed in the maximum bite force and masticatory muscle activity between the non-dysphagia and dysphagia groups (Table 1).

\section{Maximum bite force}

The average maximum bite force was $46.1 \mathrm{~N}$ (SD, $19.6 \mathrm{~N}$ ) in the dysphagia group and 86.2 N (SD, $36.3 \mathrm{~N}$ ) in the non-dysphagia group; the value was statistically much lower in the dysphagia group than in the non-dysphagia group $(P=0.024)$.

\section{Muscle activity}

The average muscle activity (MVC\%) in the temporal and masseter muscles during 1 chewing cycle was $52.0 \%$ (SD, 20.8\%) in the dysphagia group and $33.6 \%$ (SD, 15.6\%) in the non-dysphagia group; the activity was statistically much greater in the dysphagia group than in the nondysphagia group $(P=0.048)$.

\section{Number of foods items that were considered as easy to chew}

The average number of food items that were considered as easy to chew was 12.7 (SD, 2.3) in the dysphagia group and 14.5 (SD, 2.2) in the nondysphagia group. However, the difference in this factor between the 2 groups was not statistically significant.

\section{Occlusal contact area}

The average occlusal contact area in the dysphagia group was $5.4 \mathrm{~mm}^{2}\left(\mathrm{SD}, 7.0 \mathrm{~mm}^{2}\right)$ and that in the non-dysphagia group was $7.4 \mathrm{~mm}^{2}$ (SD, 5.2 $\left.\mathrm{mm}^{2}\right)$; the difference in this factor between the 2 groups was not statistically significant.

Proportion of patients with anterior open bite Anterior open bite was exhibited by 4 of the 12 patients in the non-dysphagia group and 4 of the 6 patients in the dysphagia group (Table 2).

\section{Discussion}

Although the evaluation of the masticatory function necessitated the use of some methods, the present results prove that only maximum bite force and masticatory muscle activity are relevant to dysphagia. Previous reports ${ }^{4,5}$ have indicated that $\mathrm{MyD}$ patients compensate for their weak bite force by considerably increasing their masticatory muscle activity while chewing. Based on this opinion, it is considered that the dysphagia group probably chewed with a high masticatory muscle activity because of their smaller bite force. A poor masticatory function reduces the ability to form a bolus and may also influence dysphagia. However, the cause of dysphagia may be weakness of the laryngeal muscles and not a 
poor masticatory function. The abovementioned cause of dysphagia is only an inference, and a more detailed investigation is required to draw a conclusion. A study with more number of subjects may surface the relationship between the other factors of masticatory function and dysphagia.

The Occlusal Force Meter was bitten on at the first molar region bilaterally; however, the reproducibility of the bite force level with this method has not been assessed. ${ }^{6}$ It is also well known that reproducibility of the surface electromyography signal strongly depends on electrode location, the manner in which the electrodes are repositioned, and the operator's calibration skills. ${ }^{9}$ Hence, a Dental Prescale sheet will interfere with the stability of the intercuspal position and mandibular closing paths. ${ }^{10}$ Despite the aforementioned experimental errors associated with the measurement devices used, these methods are sufficiently useful in the easy measurement of the approximate value of each masticatory factor in the $\mathrm{MyD}$ patients. These experimental errors and the small number of subjects may be considered as limitations of this study, but the results obtained in this study may indirectly imply that the bite force and occlusion also influence dysphagia in MyD patients.

To divide the MyD patients into non-dysphagia and dysphagia groups, the medical record of each patient was analyzed, and the existence of coughs or chokes during meal was regarded as dysphagia in cases wherein the patient did not undergo videofluorography. Information on the content of dysphagia was limited; therefore, when measuring the muscle activity, a cotton roll was used instead of a food item to prevent the risk of aspiration. A cotton roll was a false food item in this study, and chewing the cotton roll may be different from chewing food. Therefore, the result of the muscle activity measurement should be assessed after considering this difference.

Attending physicians selected a diet type for the MyD patients after considering their dysphagia status. From the dental point of view, however, it is doubtful whether food is chewed sufficiently during the anterior phase of deglutition. Indeed, in this study, the dysphagia group showed a higher ratio of patients with anterior open bite to all patients than the non-dysphagia group. In previous studies, ${ }^{11,12}$ a higher frequency of anterior open bite among MyD patients was reported. Kiliaridis et $\mathrm{al}^{11}$ hypothesized that a reduced masticatory muscle function may cause changes in the craniofacial morphology (e.g., anterior open bite, lateral cross bite).

Esophageal symptoms have been previously mentioned in the initial descriptions of MyD by Steinert in $1909 .{ }^{13}$ Since then, several reports have evaluated the pharyngo-esophageal motor involvement in MyD patients. ${ }^{14-16}$ Willing et $\mathrm{al}^{16}$ reported that MyD patients had many complaints related to the posterior phase of swallowing, such as choking, but fewer patients with $\mathrm{MyD}$ resorted to a complete modification of food texture when compared to the number of patients with other neuromuscular disorders. Their result about food texture is inconsistent with that of the masticatory disturbances in MyD patients; the contradictory results might be attributed to the questionnaire used in their study. The uncomplaining nature of $\mathrm{MyD}$ patients ${ }^{17}$ may create a gap between very few subjective symptoms in patients and their actual poor condition in mastication.

Among some of the methods used to assess the masticatory function, bite force and questionnaire are considered as methods convenient for attending physicians and nurses. By establishing a cut-off point (for example, $100 \mathrm{~N}$ as the maximum bite force or 16 food items as the number of foods that are considered easy to chew), patients with a poor masticatory function as well as denture wearers can be identified. For obtaining information about the masticatory function and for considering both masticatory disturbance and dysphagia, hospitals could supply appropriate diet types to MyD patients.

\section{Conclusion}

These results suggest that the bite force and anterior open bite also influence dysphagia in $\mathrm{MyD}$ patients. It is necessary to consider masticatory disturbances in $\mathrm{MyD}$ patients with dysphagia.

Acknowledgments: The authors are indebted to Dr. Kawai M, Department of Neurology at the National Center of Neurology and Psychiatry, Musashi Hospital, for enabling the examination of the MyD patients. 


\section{References}

1. Friedman RD, Joe J, Bodak LZ. Myotonic dystrophy: report of a case. Oral Surg Oral Med Oral Pathol 50: 229-232, 1980.

2. Peñarrocha M, Bagán JV, Vilchez J et al. Oral alterations in Steiner's myotonic dystrophy: a presentation of two cases. Oral Surg Oral Med Oral Pathol 69: 698-700, 1990.

3. Engvall M, Kiliaridis S, Mejersjö C. Dental needs of patients with myotonic dystrophy. Swed Dent J 15: 171-178, 1991.

4. Umemoto G, Tsukiyama Y, Nakamura H et al. Characterization of masticatory function in patients with myotonic dystrophy. - Part 1. Correlations among the factors of masticatory function-. Prosthodont Res Pract 5: 31-36, 2006.

5. Umemoto G, Tsukiyama Y, Nakamura H et al. Characterization of masticatory function in patients with myotonic dystrophy-Part 2. Comparison between patients with myotonic dystrophy and healthy individuals. Prosthodont Res Pract 5: 68-71, 2006.

6. Saifuddin MD, Miyamoto K, Ueda HM et al. A quantitative electromyographic analysis of masticatory muscle activity in usual daily life. Oral Dis 7: 94-100, 2001.

7. Schindler HJ, Stengel E, Spiess WE. Feedback control during mastication of solid food textures. - a clinical-experimental study-. J Prosthet Dent 80: 330-336, 1998.

8. Sato Y, Minagi S, Akagawa Y et al. An evaluation of chewing function of complete denture wearers.
J Prosthet Dent 62: 50-53, 1989.

9. Castroflorio T, Icardi K, Torsello F et al. Reproducibility of surface EMG in the human masseter and anterior temporalis muscle areas. Cranio 23: 130-137, 2005.

10. Matsui Y, Ohno K, Michi K et al. A computerized method for evaluating balance of occlusal load. J Oral Rehabil 23: 530-535, 1996.

11. Kiliaridis S, Mejersjö C, Thilander B. Muscle function and craniofacial morphology: a clinical study in patients with myotonic dystrophy. Eur J Orthod 11: 131-138, 1989.

12. Gazit E, Bornstein N, Lieberman $\mathrm{M}$ et al. The stomatognathic system in myotonic dystrophy. Eur $\mathrm{J}$ Orthod 9: 160-164, 1987.

13. Steinert H. Myophathologische Beiträge. I. Uber das Klinische und anatomische Bild des Muskelschwunds der Myotoniker. Dtsch Z Nervenheilk, 37: 58-104, 1909.

14. Ertekin C, Yüceyar N, Aydoğdu İ et al. Electrophysiological evaluation of oropharyngeal swallowing in myotonic dystrophy. J Neurol Neurosurg Psychiatry 70: 363-371, 2001.

15. Constantini M, Zaninotto G, Anselmino M et al. Esophageal motor function in patients with myotonic dystrophy. Dig Dis Sci 41: 2032-2038, 1996.

16. Willing TN, Paulus J, Lacau Saint Guilty J et al. Swallowing problems in neuromuscular disorders. Arch Phys Med Rehabil 75: 1175-1181, 1994.

17. Harper PS. Myotonic Dystrophy-the clinical picture. In: Harper PS, editor. Myotonic Dystrophy, 2nd ed. 13-36, London: Saunders, 1989. 